\title{
Efficacy of Preoperative Color Doppler Sonography of Lower Extremity Veins on Postoperative Outcomes in Candidates of Saphenectomy: A Randomized Clinical Trial
}

Rouhollah Zarepur ${ }^{1}$, Saeed Kargar ${ }^{1}$, Mehdi Hadadzadeh², Nooshin Hatamizadeh ${ }^{3}$, Ehsan Zarepur ${ }^{4}$, Seyed Khalil Forouzannia $^{5}$, Reza Faraji ${ }^{6}$, Mohammadtaghi Sarebanhassanabadi ${ }^{7}$

${ }^{1}$ M.D., Department of General Surgery, Faculty of Medicine, Shahid Sadoughi University of Medical Sciences, Yazd, Iran

${ }^{2}$ M.D., Cardiac Surgeon, Assistant Professor, Yazd Cardiovascular Research Center, Shahid Sadoughi University of Medical Sciences, Yazd, Iran

${ }^{3}$ M.D., Department of Obstetrics and Gynecology, Faculty of Medicine, Shahid Sadoughi University of Medical Sciences, Yazd, Iran

${ }^{4}$ M.D., Medical Student, Student Research Committee, Faculty of Medicine, Shahid Sadoughi University of Medical Sciences, Yazd, Iran

${ }^{5}$ M.D., Cardiac Surgeon, Full Professor, Tehran Heart Center, Tehran University of Medical Sciences, Tehran, Iran

${ }^{6}$ Ph.D. Student, Preventive Cardiovascular Research Centre, Kermanshah University of Medical Sciences, Kermanshah, Iran

${ }^{7}$ Ph.D. Student, Yazd Cardiovascular Research Center, Shahid Sadoughi University of Medical Sciences, Yazd, Iran

Type of article: Original

\begin{abstract}
Background: Doppler sonography is a type of sonography used for imaging the blood flow in the vessels and heart. This technique uses ultrasound waves with high frequency. In some patient candidates for venous graft, the identification of the suitable vein is not possible with clinical examination.

Objective: This study compared the effects of preoperative color Doppler sonography of lower extremity veins on the postoperative outcomes of saphenectomy.

Methods: This randomized clinical trial was conducted on 100 candidates of an off-pump coronary artery bypass graft (CABG) hospitalized in Afshar Hospital in Yazd in 2015. Patients were divided into two groups: 50 patients in the study group and 50 patients in the control group. Patients in the study group underwent color Doppler sonography of lower extremity veins using the Medison 8000 Live device. Patients in the control group were assessed preoperatively by routine venous examination without undergoing color Doppler sonography. The prepping and draping methods and also the preoperative antibiotics were the same for both groups. The patients were assessed for wound infection, edema, hematoma, and DVT 2 days, 1 week, and 1 month after surgery. Data were analyzed by SPSS version 16 using t-test, Chi-square, and Fisher's exact test.

Results: The length of incision for saphenectomy was $29.20 \pm 3.71 \mathrm{~cm}$ in the Doppler group and $28.98 \pm 3.72 \mathrm{~cm}$ in the non-Doppler group with no significant difference between the two groups $(\mathrm{p}=0.768)$. The two groups were not significantly different with respect to age, gender, diabetes, hypertension, hyperlipidemia, smoking, and history of peripheral vessels disease, postoperative infection, postoperative organ edema, postoperative hematoma, and postoperative DVT.

Conclusion: Preoperative color Doppler sonography of the saphenous vein before saphenectomy has no effect on reducing the postoperative complications, and saphenectomy on the basis of intraoperative examination of the vein course by the surgeon has acceptable consequences.

Clinical trial registration: The trial was registered at the Thai Clinical Trials Registry (TCTR) (http://www.clinicaltrials.in.th) with the TCTR ID: TCTR20160708001.

Funding: The authors received no financial support for the research, authorship, and/or publication of this article. Keywords: Doppler sonography, Saphenous vein, CABG
\end{abstract}

\section{Corresponding author:}

Professor Dr. Seyed Khalil Forouzannia, Tehran Heart Center, Tehran University of Medical Sciences, Tehran, Iran. Tel: +98.9131524497, Fax: +98.3535231421, Email: drforouzan_nia@yahoo.com

Received: May 21, 2016, Accepted: July 23, 2016, Published: September 2016

iThenticate screening: July 23, 2016, English editing: August 01, 2016, Quality control: August 06, 2016

(C) 2016 The Authors. This is an open access article under the terms of the Creative Commons Attribution-NonCommercialNoDerivs License, which permits use and distribution in any medium, provided the original work is properly cited, the use is non-commercial and no modifications or adaptations are made. 


\section{Introduction}

Various methods are used to examine vessels, while color Doppler sonography is preferred over other methods for numerous reasons. Doppler sonography consists of integration of B-mode images and Doppler wave form analysis. The old systems of Doppler sonography were limited to conventional black-and-white images; however, the possibility of creating color images has promoted the Doppler sonography to a powerful device in assessing vascular disturbances (1). The most common venous graft for CABG is the use of GSV. The saphenous graft is also used for other surgeries; yet, due to the unpredictable idiopathic morphology of the vein and the need for a long incision, many complications may result, including wound infection, hematoma, edema, and reduced mobility. To prepare the saphenous vein, a surgical incision is made along its long course, which may lead to various major problems for the patient. Incomplete healing of the wound most often causes a long hospital stay for the patient and increased costs. Various studies have reported various complications of lower extremity wound at the site of the great saphenous vein in $2 \%$ to $24 \%$ of cases $(2,3)$. To reduce the complications of lower extremity venous graft excision, the extent of the incision on the organs could be reduced to a minimum by an exact examination of the veins before surgery. Nonetheless, in most patients, the identification of the suitable vein is not possible with clinical examination. Hence, the right incision can be made at the suitable site of the lower extremities by the use of color Doppler sonography leading to the least trauma sustained by the patient $(4,5)$. In this study, the study group underwent color Doppler sonography of lower extremities as treatment, and the control group did not undergo this procedure as a control (placebo). At the end, the extent of the incision required for venous graft excision and the related complications were determined. In this way, the effect of preoperative color Doppler sonography for improving the outcomes could be determined.

\section{Material and Methods}

\subsection{Trial design and participants}

This randomized clinical trial was carried out on 100 candidates of off-pump CABG in Afshar Hospital in Yazd in 2015. The participants were divided into two groups: study group (50 patients) and control group (50 patients).

\subsection{Selection criteria}

These patients were examined in the lower extremities for varix, history of venous excision, history of thrombophlebitis, and previous severe trauma. Patients with any of these problems were excluded from the study.

\subsection{Interventions}

Fifty patients in the study group underwent venous color Doppler sonography using Medison 8000 Live, and the vein suitable for excision (a vein with appropriate diameter without branches or with a few branches) was marked. Patients in the control group were assessed preoperatively for the veins of the lower extremities using a routine clinical examination without Doppler sonography, and the incision was made intraoperatively at the suitable site on the basis of clinical findings, and the suitable graft was used. Prepping and draping methods along with the preoperative antibiotic administration were the same for both groups. At the end, patients in both groups were compared with respect to the extent of incision in the lower extremities and assessed for wound infection, edema, hematoma, and DVT at 2 days, 1 week, and 1 month after surgery.

\subsection{Outcomes}

Patients in both groups were compared with respect to the extent of incision in the lower extremities and assessed for wound infection, edema, hematoma, and DVT at 2 days, 1 week, and 1 month after surgery.

\subsection{Statistical methods, sample size, and randomization}

According to the confidence level of $95 \%$, test power of $80 \%, d=7$ and $\mathrm{S}=14$, for each group 50 patients are calculated. The samples were divided into two groups using a random numbers table. All the gleaned data were analyzed using SPSS version 16 (SPSS Inc. Chicago, Illinois, USA). The culled data were statistically analyzed using t-test, chi-square, and Fisher's exact test.

\subsection{Research ethics}

The proposal of the study was approved by the Ethics Committee of Shahid Sadoughi University of Medical Sciences. Color Doppler sonography is a noninvasive method, thus the study was done without any physical and psychological harm to the intervention group. Also, consent forms were taken for all patients. The trial was registered at the Thai Clinical Trials Registry (TCTR) (http://www.clinicaltrials.in.th) with the TCTR ID: TCTR20160708001. 


\section{Results}

This clinical trial was carried out on 100 candidates of off-pump CABG in Afshar Hospital in Yazd, central Iran, in 2015. The patients were divided into two groups: study group (50 patients) and control group (50 patients). The patients in the study group underwent color Doppler sonography before excision of the saphenous vein. Patients in the control group, however, underwent saphenectomy without Doppler sonography. The mean age of the patients in the Doppler group was 61.60 years while that of the control patients was 60.9 years. The two groups were not significantly different regarding age $(\mathrm{p}=0.716)$. Also, 26 patients $(52 \%)$ were male in the Doppler group and 24 patients were female. Moreover, in the non-Doppler group, 35 patients were male while 15 patients were female; thus the two groups were not significantly different with respect to gender $(\mathrm{p}=0.065)$. Additionally, 25 patients $(50 \%)$ in the Doppler group and 14 patients $(28 \%)$ in the non-Doppler group had diabetes, indicating no statistically significant difference between the two groups $(\mathrm{p}=0.024)$. The results of the investigation of hypertension revealed that 30 patients $(60 \%)$ in the Doppler group and 23 patients $(46 \%)$ in the non-Doppler group were affected with hypertension showing no significant difference between the two groups in this regard $(p=0.161)$. There was no significant difference between the two groups regarding hyperlipidemia, smoking, and history of peripheral vessels disorder ( $\mathrm{p}>0.05)$. Moreover, three patients $(6 \%)$ in the Doppler group and four patients $(8 \%)$ in the non-Doppler group developed infection following saphenectomy with no statistically significant difference between the two groups in this regard $(\mathrm{P}=1.000)$. The results of the study of edema demonstrated that six patients $(12 \%)$ in the Doppler group and four patients (8\%) in the non-Doppler group developed lower extremity edema after saphenectomy. So the two groups were not significantly different with respect to postoperative edema, either $(\mathrm{p}=0.741)$. Also, one patient $(2 \%)$ in the Doppler group and two patients $(4 \%)$ in the non-Doppler group developed postoperative hematoma after saphenectomy, indicating no significant difference between the two groups with regard to postoperative hematoma $(\mathrm{p}=1.000)$. Finally, one patient $(2 \%)$ in the Doppler group and two patients $(4 \%)$ in the non-Doppler group developed postoperative DVT after saphenectomy, indicating no significant difference between the two groups with regard to postoperative DVT $(\mathrm{p}=1.000)$. The length of incision for saphenous vein excision was $29.20 \pm 3.71 \mathrm{~cm}$ in the Doppler group and $28.98 \pm 3.72 \mathrm{~cm}$ in the non-Doppler group, suggesting no significant difference between the two groups in this respect, either $(\mathrm{p}=0.768)$ (Table 1).

Table 1. Estimation and comparison of variables under study in two groups

\begin{tabular}{|c|c|c|c|}
\hline \multirow{2}{*}{\multicolumn{2}{|c|}{ Variables }} & \multicolumn{2}{|c|}{ Doppler sonography; $\mathrm{n}(\%)$} \\
\hline & & Yes & No \\
\hline \multirow[t]{2}{*}{ Gender } & Male & $26(52)$ & $35(70)$ \\
\hline & Female & $24(48)$ & $15(30)$ \\
\hline \multirow[t]{2}{*}{ Diabetes } & Positive history & $25(50)$ & $14(28)$ \\
\hline & Negative history & $25(50)$ & $36(72)$ \\
\hline \multirow[t]{2}{*}{ Hypertension } & Positive history & $25(50)$ & $14(28)$ \\
\hline & Negative history & $25(50)$ & $36(72)$ \\
\hline \multirow[t]{2}{*}{ Wound infection } & Yes & $3(6)$ & $4(8)$ \\
\hline & No & $47(94)$ & $46(92)$ \\
\hline \multirow[t]{2}{*}{ Edema } & Yes & $6(12)$ & $4(8)$ \\
\hline & No & $44(88)$ & $46(92)$ \\
\hline \multirow[t]{2}{*}{ Hematoma } & Yes & $1(2)$ & $2(4)$ \\
\hline & No & $49(98)$ & $48(96)$ \\
\hline \multirow[t]{2}{*}{ DVT } & Yes & $1(2)$ & $2(4)$ \\
\hline & No & $49(98)$ & $48(96)$ \\
\hline
\end{tabular}

\section{Discussion}

Very few studies similar to ours have been carried out in the world. This study was conducted on 100 candidates of off-pump CABG who were assigned to a study group (50 patients) and a control group (50 patients). The patients in the study group underwent color Doppler sonography before saphenectomy, while those in the control group underwent saphenectomy without Doppler sonography. Our findings indicated that the mean age of patients was 61.60 years in the Doppler group and 60.90 years in the non-Doppler group. Also, there was no significant difference between the two groups in this study with respect to age, gender, diabetes, hypertension, hyperlipidemia, smoking, history of peripheral vessels disease, postoperative infection, postoperative hematoma, postoperative edema, and postoperative DVT. To prepare the patient for saphenectomy, the surgical incision is made in a long course. This brings about many complications for the patient. Incomplete healing of the incision most often leads to 
a patient's increased hospital stay and costs. Also, some complications of the lower extremity wound at the site of excision of the great saphenous vein have been reported in $2 \%-24 \%$ of cases. The study by Linni et al. (2012) conducted on 130 patients demonstrated that preoperative duplex vein mapping (DVM) decreased the costs of surgical site infections (SSI) in patients undergoing infrainguinal bypass (5). Moreover, Teixeira et al. (2015) concluded that the technique used in saphenectomy for infrainguinal arterial bypass is not correlated with postoperative SSI (6). In the present study, three patients (6\%) in the Doppler group and four patients (8\%) in the non-Doppler group developed postoperative infection after saphenectomy, indicating no significant difference between the two groups with regard to postoperative infection $(\mathrm{p}=1.000)$. Consequently, there was no statistically and clinically significant difference between the two groups with respect to wound infection. Edema is an obvious clinical problem after excision of the lower extremity saphenous vein. There are various strategies for reducing this edema, including acupressure. In our study, six patients (12\%) in the Doppler group and four patients (8\%) in the non-Doppler group developed postoperative edema of the lower extremities after saphenectomy. In this study, one patient $(2 \%)$ in the Doppler group and two patients $(4 \%)$ in the non-Doppler group developed postoperative hematoma after saphenectomy. Moreover, one patient (2\%) in the Doppler group and two patients $(4 \%)$ in the nonDoppler group developed postoperative DVT after excision of the saphenous vein. None of similar studies (comparing two groups) investigated edema, hematoma, and DVT. Broughton et al. (2013) found in their study that mapping of the saphenous vein preoperatively by sonography reduced the complications of a lower extremity wound, resulting in decreased time of saphenectomy, smaller scar, and reduced unnecessary incision (7). In this study, the length of incision for excision of the saphenous vein was $29.20 \pm 3.71 \mathrm{~cm}$ in the Doppler group and 28.98 $\pm 3.72 \mathrm{~cm}$ in the non-Doppler group, indicating no significant difference between the two groups in this respect. It appears that a study needs to be done to investigate the effectiveness of Doppler sonography (as an adjunct procedure, not as a substitute) before identifying the site of the vein by the surgeon. Further, Abu-Omar et al. (2004) showed that Doppler sonography can be helpful in determining the suitable artery in patients who are candidates of radial arterectomy and whose Allen testis was impaired (8). Moreover, Cohn et al. (2005) concluded that intraoperative Doppler sonography is effective in the selection of proper site for incision to avoid unnecessary dissection of tissue, temporal latency, destruction of the vein, and wound complications (9). The results of this study are not consistent with our findings. Our results, nevertheless, indicate that preoperative Doppler sonography before excision of the saphenous vein has no significant effect on reducing the adverse after-effects of saphenectomy and the excision of the vein on the basis of intraoperative clinical examination of the vein course by a skilled surgeon brings up acceptable outcomes. The positive point in regards to this study is RCT design and enough participants. The authors suggest using other methods such as endovascular procedure in future studies.

\section{Conclusions}

There was no significant difference between the two groups in edema, wound infection, hematoma, and length of incision. On the basis of our findings, it could be concluded that preoperative color Doppler sonography of the saphenous vein has no significant effect on reducing the postoperative complications. In spite of the availability of various advanced paraclinical devices, the clinical examination is still a valuable method and excision of the vein on the basis of intraoperative clinical examination by an expert surgeon would lead to acceptable and satisfactory results.

\section{Acknowledgments:}

The authors of this article give their special thanks to Afshar Hospital and Shahid Sadoughi University of Medical Sciences, Yazd, Iran.

\section{Clinical trial registration:}

The trial was registered at the Thai Clinical Trials Registry (TCTR) (http://www.clinicaltrials.in.th) with the TCTR ID: TCTR20160708001.

\section{Funding:}

The authors received no financial support for the research, authorship, and/or publication of this article.

\section{Conflict of Interest:}

There is no conflict of interest to be declared.

\section{Authors' contributions:}

All authors contributed to this project and article equally. All authors read and approved the final manuscript. 


\section{References:}

1) Brunicardi FC, Andersen DK, Billiar TR, Dunn DL, Hunter JG, Matthews JB, et al. Schwartzs principles of surgery, tenth Edition. 2015; 915.

2) Horvath KD, Gray D, Benton L, Hill J, Swanstrom LL. Operative outcomes of minimally invasive saphenous vein harvest. Am J Surg. 1998; 175(5): 391-5. doi: 10.1016/S0002-9610(98)00044-0. PMID: 9600285.

3) Luckraz H, Lowe J, Pugh N, Azzu A. Pre-operative long saphenous vein mapping predicts vein anatomy and quality leading to improved post-operative leg morbidity. Interact Cardiovasc Thorac Surg. 2008; 7(2): 188-91. discussion 191. doi: 10.1510/icvts.2007.166645. PMID: 18203766.

4) Galeandro AI, Quistelli G, Scicchitano P, Gesualdo M, Zito A, Caputo P, et al. Doppler ultrasound venous mapping of the lower limbs. Vasc Health Risk Manag. 2012; 8: 59-64. doi: 10.2147/VHRM.S27552. PMID: 22371652, PMCID: PMC3282606.

5) Linni K, Aspalter M, Mader N, Butturini E, Ugurluoglu A, Granat S, et al. Preoperative Duplex Vein Mapping (DVM) Reduces Costs in Patients Undergoing Infrainguinal Bypass Surgery: Results of a Prospective Randomised Study. Eur J VascEndovasc Surg. 2012; 43(5): 561-6. doi: 10.1016/j.ejvs.2012.01.030. PMID: 22386385.

6) Teixeira PG, Woo K, Weaver FA, Rowe VL. Vein harvesting technique for infrainguinal arterial bypass with great saphenous vein and its association with surgical site infection and graft patency. J Vasc Surg. 2015; 61(5): 1264-71. doi: 10.1016/j.jvs.2014.12.049. PMID: 25659457.

7) Broughton JD, Asopa S, Goodwin AT, Gildersleeve S. Could routine saphenous vein ultrasound mapping reduce leg wound complications in patients undergoing coronary artery bypass grafting? Interact Cardiovasc Thorac Surg. 2013; 16(1): 75-8. doi: 10.1093/icvts/ivs334. PMID: 23044343, PMCID: PMC3523617.

8) Abu-Omar Y, Mussa S, Anastasiadis K, Steel S, Hands L, Taggart DP. Duplex ultrasonography predicts safety of radial artery harvest in the presence of an abnormal Allen test. Ann Thorac Surg. 2004; 77(1): 116-9. doi: 10.1016/S0003-4975(03)01515-7. PMID: 14726046.

9) Cohn JD, Korver KF. Optimizing saphenous vein site selection using intraoperative venous duplex ultrasound scanning. Ann Thorac Surg. 2005; 79(6): 2013-7. doi: 10.1016/j.athoracsur.2004.12.022. PMID: 15919301. 